\title{
Transcriptional profiles reveal deregulation of lipid metabolism and inflammatory pathways in neurons exposed to palmitic acid
}

\section{Manuel Flores Leon}

UNAM Instituto de Investigaciones Biomedicas: Universidad Nacional Autonoma de Mexico Instituto de Investigaciones Biomedicas

Nicolas Alcaraz Millman

Instituto Nacional de Medicina Genómica: Instituto Nacional de Medicina Genomica

\section{Martha Perez Dominguez}

UNAM Instituto de Investigaciones Biomedicas: Universidad Nacional Autonoma de Mexico Instituto de Investigaciones Biomedicas

\section{Karla Marisa Torres Arciga}

INCan: Instituto Nacional de Cancerologia

\section{Rosa Rebollar Vega}

Instituto Nacional de Ciencias Médicas y Nutrición Salvador Zubiran: Instituto Nacional de Ciencias

Medicas y Nutricion Salvador Zubiran

Inti Alberto De la Rosa Velazquez

Helmholtz Zentrum München Deutsches Forschungszentrum für Umwelt und Gesundheit: Helmholtz Zentrum Munchen Deutsches Forschungszentrum fur Gesundheit und Umwelt

\section{Cristian Arriaga Canon}

INCan: Instituto Nacional de Cancerologia

\section{Luis A Herrera}

Instituto Nacional de Medicina Genómica: Instituto Nacional de Medicina Genomica

\section{Clorinda Arias}

UNAM Instituto de Investigaciones Biomedicas: Universidad Nacional Autonoma de Mexico Instituto de Investigaciones Biomedicas

Rodrigo González Barrios ( $\nabla$ rodrigop@ciencias.unam.mx )

INCan: Instituto Nacional de Cancerologia https://orcid.org/0000-0001-8982-6586

\section{Research Article}

Keywords: Palmitic acid, RNAseq, hippocampal neurons, neuroinflammation, lipid metabolism 
DOl: https://doi.org/10.21203/rs.3.rs-319265/v1

License: (c) (1) This work is licensed under a Creative Commons Attribution 4.0 International License. Read Full License 


\section{Abstract}

The effects of the consumption of high-fat diets (HFD) have been studied to unravel the molecular pathways they are altering in order to understand the link between increased caloric intake, metabolic diseases, and the risk of cognitive dysfunction. The saturated fatty acid, palmitic acid (PA), is the main component of HFD and it has been found increased in the circulation of obese and diabetic people. In the central nervous system, PA has been associated with inflammatory responses in astrocytes, but the effects on neurons exposed to it have not been largely investigated. Given that PA affect a variety of metabolic pathways, we aimed to analyze the transcriptomic profile activated by this fatty acid to shed light on the mechanisms of neuronal dysfunction. In the current study, we profiled the transcriptome response after PA exposition at non-toxic doses in primary hippocampal neurons. Gene ontology and Reactome pathway analysis revealed a pattern of gene expression which is associated with inflammatory pathways, and importantly, with the activation of lipid metabolism that is considered not very active in neurons. Validation by qRT-PCR of Hmgcs2, Angpt/4, Ugt8 and Rnf145 support the results obtained by RNAsEq. Overall, these findings suggest that neurons are able to respond to saturated fatty acids changing the expression pattern of genes associated with inflammatory response and lipid utilization that may be involved in the neuronal damage associated with metabolic diseases.

\section{Introduction}

The chronic consumption of high-fat diets (HFD) has been involved in the development of several pathological conditions such as obesity, type-2 diabetes and even cognitive impairments that may lead to dementia [1-5]. Different components of the HFD have been studied to unravel the molecular pathways they are altering in order to understand the link between increased caloric uptake and cellular dysfunction. The most abundant saturated-fatty acid present in the HFD is palmitic acid (PA) which was found to be increased in the circulation of obese and diabetic people [6-8]. The deleterious effects of the exposure to PA have been extensively studied in peripheral tissues; mainly in the liver, muscle and pancreas [9]. The reported effects have been associated with insulin resistance, endoplasmic reticulum stress, mitochondrial dysfunction and systemic inflammation [10-13]. Few studies have addressed the mechanisms for such deleterious effects, using transcriptome studies. For instance, in cultured human hepatocytes, genes linked to lipid transport, lipogenesis, lipid droplet growth, glucose and fatty acid metabolism have been shown to be upregulated after PA exposure [14]. Similar results were obtained in primary cultures of human pancreatic islets, where genes related to glucose and lipid metabolism were upregulated after PA exposure at non-toxic dose [15]. Another group reported the transcriptomic effect of PA in a myoblast cell line model. These authors found alterations in several pathways such as interleukin, apoptosis and insulin/PI3K signaling, among others, that are important for the ability to respond to hormones, cytokines and metabolism [16].

However, little is known regarding PA effects in neurons. This is an important question to be resolved in view of the dramatic and chronic increase in the intake of HFD and PA in modern life that has become a risk to the healthy brain function. In the brain, the astrocytes are the main brain cells responsible of fatty 
acid oxidation, but information on the metabolic responses of neurons when exposed to a high concentration of PA is still scarce $[17,18]$. Although it is generally accepted that neuronal energy demands rely exclusively in glucose oxidation and that fatty acids are not largely metabolized, recent evidence points that neurons may use both glucose and fatty acids for ATP production under specific conditions [19]. In this regard, a study with hypothalamic neurons has demonstrated that they are able to sense and metabolize long-chain free fatty acids to produce ATP by mitochondrial ß-oxidation. The increased cellular levels of ATP closes the $\mathrm{K}_{\text {ATP }}$ channels causing neuronal depolarization, involved in the hypothalamic control of energy balance of the body [20]. Furthermore, in hippocampal neurons and in differentiated human neuroblastoma cells, PA induces a reduction of the NAD ${ }^{+} / \mathrm{NADH}$ ratio, and the activity and expression of the energy sensing molecule, Sirtuin-1, is compromised and insulin resistance is generated [21, 22]. These reported effects strongly suggest the metabolization of fatty acids by neurons under specific stress conditions, such as high concentrations of PA.

To our knowledge there is no reported evidence about the transcriptomic response and characterization of how the cellular program of a neuron integrates the pleiotropic effects of an exposure to PA. In the current study, we looked for the response in gene transcription after neuronal exposure to PA in non-toxic doses. By generating transcription profiles through an RNA sequencing approach, we observe the signaling pathways and neuronal responses that are consequently altered by the exposure to this saturated fatty acid.

\section{Methods}

\section{Cell Culture and PA Treatment}

Primary hippocampal neuronal cultures were obtained from Wistar rat brains obtained from 17-day-old embryos as previously reported [22]. Animals were handled with all precautions necessary to diminish their suffering consistent with the Regulations for Research in Health Matters (México) and with the approval of the local Animal Care Committee. Briefly, hippocampi were dissected, minced with a scalpel in Krebs solution (121 mM $\mathrm{NaCl}, 4.8 \mathrm{mM} \mathrm{KCl}, 1.2 \mathrm{mM} \mathrm{KH}_{2} \mathrm{PO}_{4}, 25.4 \mathrm{mM} \mathrm{NaHCO}_{3}, 14.2 \mathrm{mM}$ Glucose, $0.004 \mathrm{mM}$ Phenol Red) and incubated with $0.25 \%$ trypsin at $37{ }^{\circ} \mathrm{C}$ for $10 \mathrm{~min}$. The hippocampi were mechanically dissociated using a cell strainer (Corning ${ }^{\circledR}$ ), and the cellular suspension was homogenized in neurobasal medium (Gibco 21103049) supplemented with 2\% B27 (Gibco 17504044), $0.5 \mathrm{mM}$ L-Glutamine (Gibco 25030-081) and $20 \mathrm{\mu g} / \mathrm{mL}$ penicillin/streptomycin (Gibco 15140-122). For oil red O staining, hippocampal neurons were plated at $1.97 \times 10^{5}$ cells $/ \mathrm{cm}^{2}$ density on 12 well plates; for RNA extraction cells were plated at $1.6 \times 10^{5}$ cells $/ \mathrm{cm}^{2}$ density on $60 \mathrm{~mm}$ in plastic dishes; and for immunodetection, neurons were plated at $1.97 \times 10^{5}$ cells $/ \mathrm{cm}^{2}$ density on 12 well plates with glass coverslips. Every plate and coverslip were previously coated with $10 \mu \mathrm{g} / \mathrm{mL}$ poly-L-lysine for $24 \mathrm{~h}$. 
Cytosine arabinoside $(10 \mu \mathrm{M})$ was added to cultures 3 days after plating to inhibit the growth of non-neuronal cells. The astrocyte population in these cultures is near $5 \%$ as measured by immunofluorescence against glial fibrillary acidic protein. Hippocampal neurons were used for experiments after 12 days in vitro (DIV) and were maintained at $37{ }^{\circ} \mathrm{C}$ in a humidified $5 \% \mathrm{CO}_{2} / 95 \%$ air atmosphere. After the $12 \mathrm{DIV}$, PA (Sigma-Aldrich) was added. PA was prepared as a stock solution in ethanol and the working solution was prepared the same day of use in $10 \%$ bovine serum albumin (BSA)/phosphate buffered saline (PBS) and was incubated at $37{ }^{\circ} \mathrm{C}$ for at least $2 \mathrm{~h}$ before adding it to the cell cultures.

\section{Oil Red O Staining Quantification}

Lipid droplets detection was performed by Oil Red O (Sigma-Aldrich) staining. Oil Red O was prepared as a stock solution $5 \%$ in isopropanol in constant agitation overnight at $4{ }^{\circ} \mathrm{C}$. Afterwards, the solution was filtrated through a Whatmann ${ }^{\circledR}$ Filter and a 6:4 (Oil Red O/Mili-Q water) was prepared and incubated at room temperature (RT) at least 20 minutes before its use. The solution was filtrated through a $0.2 \mu \mathrm{m}$ Millex-GP ${ }^{\circledR}$ Filter. For the Oil Red O staining, cell culture medium was removed, and the cells were washed twice with PBS and the cells were fixed immediately with PFA 4\%/PBS overnight at $4^{\circ} \mathrm{C}$. Afterwards, PFA was removed and the cells were washed with PBS and once with isopropanol $60 \%$. Then, cells were left to dry and when they were completely dry, Oil Red O stain was added and incubated for 2 hours. Finally, the Oil Red O stain was removed, and the cells were washed with bidistilled water until background staining was removed. For its quantification, pure isopropanol was added, and the Oil Red O stain was solubilized. The final solution was read in a multiplate reader at $520 \mathrm{~nm}$.

\section{RNA Extraction}

Total RNA was isolated using TRIzol ${ }^{\mathrm{TM}}$ reagent (Thermo Fisher Scientific) as specified by the manufacturer and cDNA was synthesized from 200 ng of RNA using the High Capacity cDNA Reverse Transcription Kit (Applied Biosystems/Thermo Fisher, 4374966) with random primers. The quantification of total RNA was achieved using a NanoDrop 2000 (ThemorScientific). The integrity of RNA was assessed by Agarose-Gel Electrophoresis.

\section{RNA Sequencing}

RNA was quantified using Qubit 2.0 (Invitrogen, USA) and quality was assessed using the Agilent 2100 Bioanalyzer (Agilent Technologies, USA). Only samples with an RNA Integrity Number (RIN) >8.0 were used. 
Libraries were constructed using 500 ng of RNA, using the Truseq Stranded mRNA library prep kit from Illumina according to the manufacturer's instruction. The libraries were sequenced using an Illumina HiSeq2500 equipment (Illumina, Inc.) in Pair-end (2x125 bases). Depth of sequencing was > 25 million reads.

\section{RNA-seq Analysis}

Adapter trimming and low-quality reads were filtered out using trimmomatic v.0.39 [23]. Reads were mapped to the rat genome with STAR v.2.7.1a [24], and also to the rat transcriptome with salmon v.0.14.1 [25], using assembly version Rnor6 with ensembl annotations version 6.0.95. Gene level counts from reads mapped to the genome were quantified using featureCounts in the rsubread [26] package. Differential expression analysis was performed with DEseq2 [27] and edgeR[28], with a paired design specimen + treatment. Differentially expressed genes were defined as having $\mathrm{FDR}<0.1$ and $|\log 2 \mathrm{FC}|>=0.5$. Low expressed genes were filtered out before edgeR using the filterByExpr function and the design matrix. GO-term and pathway overrepresentation were performed with the clusterProfiler [29] Bioconductor package on all genes found DE in at least one of the 4 workflows using all expressed genes as background. Gene set enrichment analysis [30] was performed on all expressed genes ordered by their DE - $\log 10$ p-value.

\section{Quantitative RT-PCR (qRT-PCR)}

It was performed using the Maxima SYBR Green/ ROX qPCR Master Mix (Thermo Scientific, K0221) in a QuantStudio 3 (Applied Biosystems). All reactions were performed five or seven times, and the expression was normalized using the glyceraldehyde-3-phosphate dehydrogenase (Gapdh) mRNA. The sequences of the primers used are listed in Table 1.

Table 1 Primer sequences that were used for gene quantification 


\begin{tabular}{cc}
\hline Gene & Sequence \\
\hline Angptl4 & F: 5'-GGACCTTAACTGTGCCAAGA-3' \\
& R: 5'-TTTTCCAGAAGATCCCCTTT-3' \\
\hline Hmgcs2 & F:5'-ACCTTGAACGAGTGGATGAG-3' \\
& R:5'-CACCGCAGAGCAGATCCTAT-3' \\
\hline \multirow{2nf145}{*}{ F: 5'-TTACAACGTGTGGCTTCGTG-3' } & R: 5'-GATCACAGCGGATTTCATGTC-3' \\
\hline \multirow{2}{*}{ ggt8 } & F: 5'-AGTACAGGCGAAAGGCATGG-3' \\
& R: 5'-ATCTGATGGACAGCCGAACG-3' \\
\hline \multirow{2}{*}{ Gapdh } & F: 5'-GCCTGGAGAAACCTGCCAA-3' \\
& R: 5'-CTTTAGTGGGCCCTCGGC -3' \\
\hline
\end{tabular}

\section{Immunofluorescence and Image Analysis}

After different PA treatments, the cell culture media was removed, and cells were washed three times with icecold PBS. Then, the cells were fixed with ice-cold PFA 1\% / PBS for 5 min and washed three times with PBS. Afterwards, cells were permeabilized in 0.3\% Triton X-100/PBS for 30 min at RT. Then, cells were incubated in blocking solution (BSA 4\% / PBS) with gentle agitation for $1 \mathrm{~h}$ at RT. Next, cells were incubated with anti-MAP2 antibody (1:1000, Millipore \#MAB378) in blocking solution for 48 hours at $4^{\circ} \mathrm{C}$. After washing three times in 0.3\% Triton X-100/PBS, cells were incubated with secondary antibody (1:1000, Alexa Fluor 488 donkey antimouse Invitrogen \# A21202) in blocking solution for $2 \mathrm{~h}$, at RT. Immediately after incubation with the secondary antibody, nuclei were stained with Hoechst (1:1000) in PBS for $10 \mathrm{~min}$ at RT. Cells were washed three times with PBS and covered with fluorescent mounting medium (DAKO). Negative controls were performed excluding the primary antibodies from the procedure. Observations were performed on a Nikon A1R+confocal microscope (Nikon Instruments Inc) with a Plan Apo 20× (N.A. 0.75) and Plan Apo 60× water (N.A. 1.2) objectives and digital images were obtained with NIS-Elements C imaging software (Nikon).

\section{Statistical Analysis}

For the gene expression and oil red experiments, a two-tailed unpaired Student's t test was performed using a GraphPad Prism version 8.0 for Windows (GraphPad Software, San Diego, California USA, www.graphpad.com). All values are expressed as mean \pm S.E.M.

\section{Results}




\section{PA exposure changes the transcriptional profile without modifying the neuronal morphology}

To characterize the morphological consequences and neurite integrity after $24 \mathrm{~h}$ of exposure to PA, we first performed a qualitative analysis of the distribution of the cytoskeletal protein MAP2 in the hippocampal neurons (Fig. 1a). As shown, the MAP2 immunodetection was located mainly in the neurites, scarcely found in the neuronal soma and we did not observe evident effects on the neuronal morphology with 100 and $200 \mu \mathrm{M}$ PA compared to the control condition. However, neurons exposed to $300 \mu \mathrm{M}$ of PA showed a slight increase in the concentration of MAP2 into the soma and a fragmented pattern in the localization of this protein, suggesting dendritic blebbing. This distribution of MAP2 is consistent with the induction of localized swelling and may indicate toxic effects of PA at concentrations above of $300 \mu \mathrm{M}$. To estimate the neuronal lipid content after PA exposure we stained neurons with Oil Red. Spectrophotometric quantification showed that PA caused an increase in the neuronal content of lipid droplets (Fig. 1b), demonstrating the uptake and metabolization of this saturated fatty acid by hippocampal neurons. Hence, considering this effect and previous viability assays [22] we continued the experiments with the dose of $200 \mu \mathrm{M}$ PA.

Given that the fatty acids per se can alter gene regulation in periphery cells and knowing that PA has pleiotropic effects, we then asked to what extent hippocampal neurons modify their transcriptomic profile after PA exposure. By RNAseq after $24 \mathrm{~h}$ of $200 \mu \mathrm{M}$ PA, since different mapping methods can have different transcript detection sensitivities [31], we mapped sequences to both the rat's genome and transcriptome. In addition, for each mapping and gene quantification method, we used two well established methods (DEseq2 and edgeR) for differential expression analyses, making a total of 4 different pipelines. This multiple analysis showed that the gene expression was not drastically altered (Fig. 2) and it revealed a total of 45 upregulated and 30 downregulated genes (Fig. 2a, b) in at least one pipeline. Genes such as Anpt/4, Ugt8, Hmgcs2, Ccl2, and Insig 1 were shared between the different pipelines (Supplementary Table 1), which suggest that these genes are strongly affected in the neurons by PA.

Clustering of differentially expressed genes (DEGs) showed that each experimental condition in a group behaves with a similar expression pattern (Fig. 2a) and suggests that the exposure to PA can cluster experimental conditions into two different groups but that between individuals, there are specific changes and alterations. Furthermore, as shown in the differential expression analysis (Fig. 2c, d), not only proteincoding genes are deregulated by this exposure but also long non-coding intergenic RNAs (LincRNAs) and small nuclear RNAs (snRNAs). All together these findings demonstrate that PA exposure is sufficient to induce changes in the transcriptional profile of neurons; suggesting that cellular mechanisms, even those involved in nuclear function, are being altered.

\section{Changes in neuronal lipid metabolism and inflammatory pathways are induced by PA}

To determine the biological processes and the DEGs that are modified after the PA exposure, we performed pathway enrichment analyses. Gene Ontology (GO) enrichment analysis (Fig. 3a) showed that among the top 20 functionally enriched biological processes the chemokine-mediated signaling pathway, 
fatty acid and chemokine responses, cholesterol biosynthetic pathway and insulin function were altered during this exposure, indicating that these processes are importantly involved in the neuronal response to PA.

In order to determine the relationships between enriched GO terms and DEGs, we constructed an interaction network using the clusterProfiler R package (Fig. 3c). We observe that the network forms a single connected component, pointing that most genes are GO-terms closely related and work together in similar processes. In this regard, Hmgcs2 and Insig 1 are part of an important node for energy metabolism since it is known they are involved in the response to fatty acid, insulin, starvation, and cholesterol biosynthesis. The interaction network also showed that genes, such as $C c / 2$, involved in the cellular response to chemokines and the chemokine-mediated signaling pathway are related to lipid metabolism. These results suggest that PA might be altering the neuronal energy metabolism, particularly the lipid metabolism, and the inflammatory response through the induction of specific genes that are linked to both processes.

To further confirm and unravel some other biological processes affected by the exposure of neurons to PA, we performed the same enrichment analysis with Reactome [32] pathways (Fig. 4). This analysis confirmed the $\mathrm{GO}$ categories previously found not only by showing metabolism of lipids and the fatty acid cycle as important responsive pathways in neurons after PA exposure, but also revealed additional deregulated processes such as ion-channel transport and, strikingly, mitochondrial fatty acid betaoxidation (Fig. 4a, b). The analysis of the interaction network showed that multiple genes involved in lipid metabolism are deregulated, such as Ugt8, Cyp51, Acot1 and Echs1, suggesting that the metabolism of lipids is one of the most relevant biological processes activated in response to PA (Fig. 4c). Furthermore, Echs 1 and Acot1, also link the lipid metabolism with the mitochondrial beta-oxidation (Fig. 4c), which portraits them as important effectors in both processes. Overall, together $\mathrm{GO}$ and Reactome enrichment analyses and the interaction networks strongly indicate that PA is affecting mainly lipid utilization pathways, which is interesting and unexpected because they are believed to be not completely active in neurons.

\section{Genes dependent on PPAR signaling and lipid metabolism are deregulated in neurons}

GO term and pathway enrichment have the disadvantage that only DEGs are considered, however other relevant processes may be driven by subtle changes in multiple genes of the same biological pathway. In order to elucidate these, we performed Gene Set Enrichment Analysis (GSEA) which takes into account the whole list genes, both on KEGG [33] pathways (Fig. 5) and GO term gene sets (Supplementary Fig. 1). First, we found that after PA exposure the IL-17, TNF and MAPK signaling pathways are deregulated (Fig. 5). Suggesting that PA triggers inflammatory components in neurons. It is also shown that the fatty acid metabolism and catabolism (Fig. 5 and Supplementary Fig. 1), PPAR signaling pathway, the synthesis of ketone bodies (Fig. 5), cholesterol biosynthetic and metabolic processes (Supplementary Fig. 1) are likewise affected. This result indicates that neurons have lipid metabolism elements that sense and respond to a high dose of saturated fatty acids. Additionally, this analysis showed that PA can affect 
general cellular and biological processes like apoptosis and cellular senescence (Fig. 5), as well as specific neuronal processes like ensheathment of neurons and axons, axonogenesis and regulation of neurotransmitter levels (Supplementary Fig. 1).

In order to validate the results obtained by the RNAseq and to show that the PPAR signaling pathway is responding to the PA stimulus, we analyzed 4 genes by qRT-PCR. Hmgsc2 and Angpt/4 are two genes implicated in lipid metabolism and regulated by the PPAR signaling pathway. Their mRNA quantification by qRT-PCR showed that both genes (Fig. 6a, b) are significantly increased in the hippocampal neurons that were exposed to $200 \mu \mathrm{M}$ PA compared to the control condition. On the other hand, Ugt8 and Rnf145, two other genes implicated in lipid metabolism but not regulated by the PPAR signaling pathway were downregulated (Fig. 6c, d). Overall, these results and the presence of lipid bodies (Fig. 1b) demonstrate that when exposed to PA, neurons modify their transcriptional profile affecting the lipid metabolism through signaling pathways that are known to respond to fatty acid stimulus in periphery cells.

\section{Discussion}

The intake of HFD has been associated with the development of metabolic diseases and to the onset of neurodegenerative diseases [34-38]. These types of diets harbor PA as one of the main saturated fatty acids [39-41]. Although several mechanisms regarding its effects have been characterized in peripheral tissues [10-13], it remains poorly understood the neuronal responses activated by PA. Despite evidence that neurons can barely able to metabolize and respond directly to saturated fatty acids, new reports have shown that these cells are affected by pathological concentrations of PA [21, 22]. Additionally, it is known that the PA has pleiotropic effects since it can bind to transcription factors, activate signaling pathways through membrane-bound or nuclear receptors, be used in energy metabolism or as a precursor in the synthesis of other molecules, among other described effects [42]. Here we showed several functional pathways and cellular processes that are altered in neurons exposed to a non-toxic but a high concentration of PA, particularly those involved in lipid metabolism, insulin signaling and inflammatory responses.

As shown in the GSEA of KEGG pathways analysis, signaling through IL-17 is one of the most sensitive pathways activated in neurons after PA exposure. This signaling pathway belongs to the proinflammatory response well characterized mainly in T-cells and macrophages [43-46], as well as in hypothalamic neurons [47]. The family of transcription factors NF-KB have been implicated in the IL-17 signaling and in the regulation of other pathways and transcription factors such as p53, MAPK and PPAR [48-51]. Interestingly, we have previously found that PA increases the acetylated form of p65 in hippocampal neurons, which might be involved in the regulation of IL-17 effects [22]. Furthermore, it has been reported that PA can also trigger inflammatory response through the Toll-Like Receptor 4 (TLR4) inducing the transcriptional activity of p65 $[52,53]$. In addition, p65 is also a central component of the inflammatory response of the inflammasome, NLRP3 [54]. It is known that the NLRP3 can not only be induced by p65 activation but also by external stimuli that can bind to the TLR4 and/or generate ER stress, mitochondrial dysfunction and ROS elevation [53-55]. In this regard, the GSEA shows that several 
of these mechanisms are being impacted in hippocampal neurons, suggesting that neurons could be responding to the presence of high PA concentrations through this inflammatory component.

On the other hand, it is known that one of the characteristics of the inflammasome response is cytoplasmic swelling due to the ion influx/efflux. Additionally, MAPK is an important signaling pathway that can be regulated by inflammatory stimuli and usually it is activated along with these inflammatory pathways; either by a direct phosphorylation of p65 by p38 or through the activation of JNK [56, 57]. Our results show that all of these pathways are altered when neurons are exposed to PA, and are significantly involved in the response to this saturated fatty acid. The inflammatory response might be correlated to the dendritic bebbling we observed when neurons were exposed to cytotoxic concentrations of PA. Thus, present results provide useful evidence about the inflammatory reaction that PA may exert on neurons giving support to the hypothesis regarding the role of PA in the induction of chronic neuronal damage.

Besides the inflammatory process, we have also found metabolic pathways that respond to the PA exposure. Previously, we have reported a diminished consumption of glucose as well as a reduction of the $\mathrm{NAD}^{+} / \mathrm{NADH}$ ratio after neuronal exposure to $\mathrm{PA}$, suggesting that this saturated fatty acid can be used as energetic fuel by the neurons [22]. Although the utilization of saturated fatty acid as energy substrates for the brain is still controversial, the results from present analysis showed that neurons can respond to PA activating diverse lipid metabolic pathways to produce energy or to synthetize several other compounds. In this regard, it is well known that the Peroxisome Proliferator-Activated Receptors (PPAR) nuclear transcription factor proteins can bind and respond to different types of lipids [58]. Particularly, it has been described in HepG2 cells that PA and the monosaturated fatty acid, oleic acid, can specifically bind and activate PPARa and/or PPARg, in a time and concentration dependent manner [59]. Furthermore, our results showed that many other metabolic pathways that respond to nutrients are also altered by PA, such as FoxO, PI3K-Akt, synthesis and degradation of ketone bodies and cholesterol metabolism. Several research groups have reported similar effects of PA in different cell models when using RNAseq or microarrays $[15,16,60-62]$. These groups show an alteration of genes involved in the PPAR signaling pathway, fatty acid degradation, chemokine signaling pathway, inflammation pathways, beta-oxidation, insulin signaling, among others. In fact, we have previously reported that PA induces insulin resistance in neurons, similarly to that produce in periphery cells [21]. Thus, current results strongly suggest that these signaling networks and cellular processes are impacted by PA in different cell types.

Several genes encoding enzymes involved in lipid and energy metabolism were evaluated with qRT-PCR. One of the metabolic pathways that is used for energy production under fasting conditions is the ketone body synthesis. In this pathway, the mitochondrial enzyme 3-hydroxy-3-methylglutaryl-CoA Synthase 2 (Hmgcs2) catalyzes the first reaction of ketogenesis and is activated in the presence of acetyl-CoA, mostly derived from mitochondrial beta-oxidation. The transcriptional control of this gene is mediated by fatty acids through PPAR binding [63]. Herein, we demonstrated that the transcript that encodes Hmgcs2 was upregulated, suggesting that this lipid-dependent pathway is being activated in the neurons. Another gene shown to be upregulated is Angpt/4. Interestingly, this gene is also a target of 
PPAR and is induced under hypoxic conditions in various cell types. The encoded protein is a serum hormone directly involved in regulating lipid metabolism [64].

Interestingly, the other two genes that we measured by qRT-PCR, which are involved in lipid metabolism but not regulated by the PPAR transcription factor family, were found to be downregulated, Ugt8 [65] and Rnf145 [66]. Ugt8 is a brain-specific-key enzyme in the last step for the synthesis of galactocerebrosides $[67,68]$. The recently characterized Rnf145 enzyme, is a E3-ubiquitin ligase involved in the homeostasis of cholesterol, negatively regulating this process when high levels of this protein are found in a cell [69, 70]. The downregulation of Rnf145 transcript suggests alterations in the cholesterol pathway leading to an increase of the cholesterol biosynthetic genes expression and the novo synthesis of cholesterol [69].

Transcriptional expression levels of different genes involved in PA effects on neurons showed a significant impact on different neuronal processes associated with apoptosis, senescence, and autophagy, that are known to participate in the pathophysiology of neurodegenerative diseases, such as Alzheimer's Disease. Therefore, this first approach using a global analysis of gene expression can help to understand the pleiotropic effects of PA on neurons and the possible pathways activated under pathological conditions.

\section{Declarations}

Funding This work was supported by CONACYT (CA, A1-S9559) (RG-B, 2900441) and partially supported by Universidad Nacional Autónoma de México (UNAM) (PAPIIT IN202615). M Flores-León is a doctoral student from Programa de Doctorado en Ciencias Bioquímicas, Universidad Nacional Autónoma de México (UNAM) and received a fellowship from Conacyt (449712). K Torres-Arciga is a master student from Posgrado en Ciencias biológicas, Universidad Nacional Autónoma de México (UNAM) and acknowledges the support from CONACYT as a beneficiary of a scholarship (CVU1009360).

Authors' contributions MFL designed and performed experiments, analyzed data, prepared the figures and drafted the manuscript; NA performed the bioinformatical data analyses, prepared figures and reviewed the manuscript; MPD prepared the neuronal cultures and assisted with the confocal analysis; KTA assisted with the qRT-PCR experiments and prepared the figures; RRV prepared the sequencing libraries and performed the RNAseq; IARV performed the RNAseq and reviewed the manuscript; CAC contributed valuably to the discussion, writing and approval of manuscript; LAH contributed to the revision of the manuscript; CA and RGB funded, designed and oversaw the whole project including experimental design, data analysis, drafting and reviewing the manuscript.

Data availability

The sequencing data is available through the Gene Expression Omnibus database. Accession number: GSE166985.

Code availability Not applicable.

Conflict of Interest The authors declare that they have no conflict of interest. 

Committee according to the Regulations for Research in Health Matters (México).

\section{Consent for Publication}

All authors have reviewed and approved the manuscript for publication in this journal.

Consent to participate Not applicable.

Acknowledgements Not applicable.

\section{References}

1. Craft S (2009) The role of metabolic disorders in Alzheimer disease and vascular dementia: Two roads converged. Arch. Neurol

2. Calvo-Ochoa E, Arias C (2015) Cellular and metabolic alterations in the hippocampus caused by insulin signalling dysfunction and its association with cognitive impairment during aging and Alzheimer's disease: studies in animal models. Diabetes Metab Res Rev 31:1-13. https://doi.org/10.1002/dmrr.2531

3. Cordner ZA, Tamashiro KLK (2015) Effects of high-fat diet exposure on learning \& memory. Physiol. Behav

4. Hoscheidt SM, Starks EJ, Oh JM et al (2016) Insulin Resistance is Associated with Increased Levels of Cerebrospinal Fluid Biomarkers of Alzheimer's Disease and Reduced Memory Function in At-Risk Healthy Middle-Aged Adults. J Alzheimer's Dis. https://doi.org/10.3233/JAD-160110

5. González-Reyes E, Aliev R, Avila-Rodrigues G, Barreto ME G (2016) Alterations in Glucose Metabolism on Cognition: A Possible Link Between Diabetes and Dementia. Curr Pharm Des. https://doi.org/10.2174/1381612822666151209152013

6. Boden G, Shulman GI (2002) Free fatty acids in obesity and type 2 diabetes: Defining their role in the development of insulin resistance and $\beta$-cell dysfunction. Eur. J. Clin. Invest

7. Delarue J, Magnan C (2007) Free fatty acids and insulin resistance. Curr. Opin. Clin. Nutr. Metab. Care

8. Kurotani K, Sato M, Ejima Y et al (2012) High levels of stearic acid, palmitoleic acid, and dihomo-Ylinolenic acid and low levels of linoleic acid in serum cholesterol ester are associated with high insulin resistance. Nutr Res. https://doi.org/10.1016/j.nutres.2012.07.004

9. Ly LD, Xu S, Choi S-K et al (2017) Oxidative stress and calcium dysregulation by palmitate in type 2 diabetes. Exp Mol Med. https://doi.org/10.1038/emm.2016.157

10. Ishii M, Maeda A, Tani S, Akagawa M (2015) Palmitate induces insulin resistance in human HepG2 hepatocytes by enhancing ubiquitination and proteasomal degradation of key insulin signaling molecules. Arch Biochem Biophys. https://doi.org/10.1016/j.abb.2014.12.009 
11. Barlow J, Jensen VH, Jastroch M, Affourtit C (2016) Palmitate-induced impairment of glucosestimulated insulin secretion precedes mitochondrial dysfunction in mouse pancreatic islets. Biochem J. https://doi.org/10.1042/BJ20151080

12. Kwon $\mathrm{CH}$, Sun JL, Jeong JH, Jung TW (2020) Humanin attenuates palmitate-induced hepatic lipid accumulation and insulin resistance via AMPK-mediated suppression of the mTOR pathway. Biochem Biophys Res Commun. https://doi.org/10.1016/j.bbrc.2020.03.128

13. Zeng X, Zhu M, Liu X et al (2020) Oleic acid ameliorates palmitic acid induced hepatocellular lipotoxicity by inhibition of ER stress and pyroptosis. Nutr Metab (Lond). https://doi.org/10.1186/s12986-020-0434-8

14. Breher-Esch S, Sahini N, Trincone A et al (2018) Genomics of lipid-laden human hepatocyte cultures enables drug target screening for the treatment of non-alcoholic fatty liver disease. BMC Med Genomics. https://doi.org/10.1186/s12920-018-0438-7

15. Hall E, Volkov P, Dayeh T et al (2014) Effects of palmitate on genome-wide mRNA expression and DNA methylation patterns in human pancreatic islets. BMC Med 12:. https://doi.org/10.1186/17417015-12-103

16. Grabiec K, Majewska A, Wicik Z et al (2016) The effect of palmitate supplementation on gene expression profile in proliferating myoblasts. Cell Biol Toxicol 32:185-198. https://doi.org/10.1007/s10565-016-9324-2

17. Edmond J, Robbins RA, Bergstrom JD et al (1987) Capacity for substrate utilization in oxidative metabolism by neurons, astrocytes, and oligodendrocytes from developing brain in primary culture. $J$ Neurosci Res. https://doi.org/10.1002/jnr.490180407

18. Lovatt D, Sonnewald U, Waagepetersen HS et al (2007) The transcriptome and metabolic gene signature of protoplasmic astrocytes in the adult murine cortex. J Neurosci. https://doi.org/10.1523/JNEUROSCI.3404-07.2007

19. Joyal JS, Sun Y, Gantner ML et al (2016) Retinal lipid and glucose metabolism dictates angiogenesis through the lipid sensor Ffar1. Nat Med. https://doi.org/10.1038/nm.4059

20. Jo YH, Su Y, Gutierrez-Juarez R, Chua S (2009) Oleic acid directly regulates POMC neuron excitability in the hypothalamus. J Neurophysiol. https://doi.org/10.1152/jn.91294.2008

21. Calvo-Ochoa E, Sánchez-Alegría K, Gómez-Inclán C et al (2017) Palmitic acid stimulates energy metabolism and inhibits insulin/PI3K/AKT signaling in differentiated human neuroblastoma cells: The role of mTOR activation and mitochondrial ROS production. Neurochem Int. https://doi.org/10.1016/j.neuint.2017.09.008

22. Flores-León M, Pérez-Domínguez M, González-Barrios R, Arias C (2019) Palmitic Acid-Induced NAD + Depletion is Associated with the Reduced Function of SIRT1 and Increased Expression of BACE1 in Hippocampal Neurons. Neurochem Res 44:1745-1754. https://doi.org/10.1007/s11064-019-028108

23. Bolger AM, Lohse M, Usadel B (2014) Trimmomatic: A flexible trimmer for Illumina sequence data. Bioinformatics. https://doi.org/10.1093/bioinformatics/btu170 
24. Dobin A, Davis CA, Schlesinger F et al (2013) STAR: Ultrafast universal RNA-seq aligner. Bioinformatics. https://doi.org/10.1093/bioinformatics/bts635

25. Patro R, Duggal G, Love Ml et al (2017) Salmon provides fast and bias-aware quantification of transcript expression. Nat Methods. https://doi.org/10.1038/nmeth.4197

26. Liao Y, Smyth GK, Shi W (2019) The R package Rsubread is easier, faster, cheaper and better for alignment and quantification of RNA sequencing reads. Nucleic Acids Res. https://doi.org/10.1093/nar/gkz114

27. Love MI, Huber W, Anders S (2014) Moderated estimation of fold change and dispersion for RNA-seq data with DESeq2. Genome Biol. https://doi.org/10.1186/s13059-014-0550-8

28. Robinson MD, McCarthy DJ, Smyth GK (2009) edgeR: A Bioconductor package for differential expression analysis of digital gene expression data. Bioinformatics. https://doi.org/10.1093/bioinformatics/btp616

29. Yu G, Wang LG, Han Y, He QY (2012) ClusterProfiler: An R package for comparing biological themes among gene clusters. Omi A J Integr Biol. https://doi.org/10.1089/omi.2011.0118

30. Subramanian A, Tamayo P, Mootha VK et al (2005) Gene set enrichment analysis: a knowledgebased approach for interpreting genome-wide expression profiles. Proc Natl Acad Sci U S A 102:15545-15550. https://doi.org/10.1073/pnas.0506580102

31. Wu DC, Yao J, Ho KS et al (2018) Limitations of alignment-free tools in total RNA-seq quantification. BMC Genom. https://doi.org/10.1186/s12864-018-4869-5

32. Jassal B, Matthews L, Viteri G et al (2020) The reactome pathway knowledgebase. Nucleic Acids Res. https://doi.org/10.1093/nar/gkz1031

33. Kanehisa M, Goto S (2000) KEGG: Kyoto Encyclopedia of Genes and Genomes. Nucleic Acids Res

34. Umegaki H (2010) Pathophysiology of cognitive dysfunction in older people with type 2 diabetes: vascular changes or neurodegeneration? Age Ageing 39:8-10. https://doi.org/10.1093/ageing/afp211

35. Schrijvers EMC, Witteman JCM, Sijbrands EJG et al (2010) Insulin metabolism and the risk of Alzheimer disease: The Rotterdam Study. Neurology 75:1982-1987. https://doi.org/10.1212/WNL.0b013e3181ffe4f6

36. Matsuzaki T, Sasaki K, Tanizaki Y et al (2010) Insulin resistance is associated with the pathology of Alzheimer disease: The Hisayama study. Neurology 75:764-770. https://doi.org/10.1212/WNL.0b013e3181eee25f

37. Benoit SC, Kemp CJ, Elias CF et al (2009) Palmitic acid mediates hypothalamic insulin resistance by altering PKC- $\theta$ subcellular localization in rodents. J Clin Invest. https://doi.org/10.1172/JCI36714

38. Contreras A, Del Rio D, Martínez A et al (2017) Inhibition of hippocampal long-term potentiation by high-fat diets: Is it related to an effect of palmitic acid involving glycogen synthase kinase-3? Neuroreport. https://doi.org/10.1097/WNR.0000000000000774 
39. Mancini A, Imperlini E, Nigro E et al (2015) Biological and nutritional properties of palm oil and palmitic acid: Effects on health. Molecules

40. Carta G, Murru E, Lisai S et al (2015) Dietary triacylglycerols with palmitic acid in the sn-2 position modulate levels of N-Acylethanolamides in rat tissues. PLoS One. https://doi.org/10.1371/journal.pone.0120424

41. Chen B, Huang Y, Zheng D et al (2018) Dietary fatty acids alter lipid profiles and induce myocardial dysfunction without causing metabolic disorders in mice. Nutrients. https://doi.org/10.3390/nu10010106

42. Korbecki J, Bajdak-Rusinek K (2019) The effect of palmitic acid on inflammatory response in macrophages: an overview of molecular mechanisms. Inflamm Res 68:915-932. https://doi.org/10.1007/s00011-019-01273-5

43. Samuels JS, Holland L, López M et al (2018) Prostaglandin E2 and IL-23 interconnects STAT3 and RoRy pathways to initiate Th17 CD4 + T-cell development during rheumatoid arthritis. Inflamm. Res

44. Machacek M, Saunders H, Zhang Z et al (2019) Elevated O-GlcNAcylation enhances proinflammatory Th17 function by altering the intracellular lipid microenvironment. J Biol Chem 294:8973-8990. https://doi.org/10.1074/jbc.RA119.008373

45. Majumder S, Amatya N, Revu S et al (2019) IL-17 metabolically reprograms activated fibroblastic reticular cells for proliferation and survival. Nat Immunol. https://doi.org/10.1038/s41590-019-03674

46. McGeachy MJ, Cua DJ, Gaffen SL (2019) The IL-17 Family of Cytokines in Health and Disease. Immunity 50:892-906. https://doi.org/10.1016/j.immuni.2019.03.021

47. Nogueira G, Solon C, Carraro RS et al (2020) Interleukin-17 acts in the hypothalamus reducing food intake. Brain Behav Immun. https://doi.org/10.1016/j.bbi.2019.12.012

48. Awane M, Andres PG, Li DJ, Reinecker HC (1999) NF-kappa B-inducing kinase is a common mediator of IL-17-, TNF-alpha-, and IL-1 beta-induced chemokine promoter activation in intestinal epithelial cells. J Immunol

49. Schneider G, Henrich A, Greiner G et al (2010) Cross talk between stimulated NF-kB and the tumor suppressor p53. Oncogene. https://doi.org/10.1038/onc.2010.46

50. An YQ, Zhang CT, Du Y et al (2016) PPARס agonist GW0742 ameliorates A $\beta 1$-42-induced hippocampal neurotoxicity in mice. Metab Brain Dis. https://doi.org/10.1007/s11011-016-9800-7

51. Fann DYW, Lim YA, Cheng YL et al (2018) Evidence that NF-KB and MAPK Signaling Promotes NLRP Inflammasome Activation in Neurons Following Ischemic Stroke. Mol Neurobiol. https://doi.org/10.1007/s12035-017-0394-9

52. Akieda-Asai S, Ma H, Date Y (2019) Palmitic acid induces guanylin gene expression through the tolllike receptor 4/nuclear factor-kB pathway in rat macrophages. Am J Physiol - Cell Physiol. https://doi.org/10.1152/ajpcell.00081.2019

53. Dong Z, Zhuang Q, Ning M et al (2020) Palmitic acid stimulates NLRP3 inflammasome activation through TLR4-NF-kB signal pathway in hepatic stellate cells. Ann Transl Med. 
https://doi.org/10.21037/atm.2020.02.21

54. Li DX, Wang CN, Wang Y et al (2020) NLRP3 inflammasome-dependent pyroptosis and apoptosis in hippocampus neurons mediates depressive-like behavior in diabetic mice. Behav Brain Res 391:112684. https://doi.org/10.1016/j.bbr.2020.112684

55. Swanson KV, Deng M, Ting JPY (2019) The NLRP3 inflammasome: molecular activation and regulation to therapeutics. Nat Rev Immunol 19:477-489. https://doi.org/10.1038/s41577-019-01650

56. Santa-Cecília FV, Socias B, Ouidja MO et al (2016) Doxycycline Suppresses Microglial Activation by Inhibiting the p38 MAPK and NF-kB Signaling Pathways. Neurotox Res. https://doi.org/10.1007/s12640-015-9592-2

57. Shrestha A, Bruckmueller $\mathrm{H}$, Kildalsen $\mathrm{H}$ et al (2020) Phosphorylation of steroid receptor coactivator3 (SRC-3) at serine 857 is regulated by the p38MAPK-MK2 axis and affects NF-KB-mediated transcription. Sci Rep. https://doi.org/10.1038/s41598-020-68219-4

58. Varga T, Czimmerer Z, Nagy L (2011) PPARs are a unique set of fatty acid regulated transcription factors controlling both lipid metabolism and inflammation. Biochim Biophys Acta - Mol Basis Dis 1812:1007-1022. https://doi.org/10.1016/j.bbadis.2011.02.014

59. Popeijus HE, van Otterdijk SD, van der Krieken SE et al (2014) Fatty acid chain length and saturation influences PPARa transcriptional activation and repression in HepG2 cells. Mol Nutr Food Res 58:2342-2349. https://doi.org/10.1002/mnfr.201400314

60. Cnop M, Abdulkarim B, Bottu G et al (2014) RNA sequencing identifies dysregulation of the human pancreatic islet transcriptome by the saturated fatty acid palmitate. Diabetes 63:1978-1993. https://doi.org/10.2337/db13-1383

61. Sargsyan E, Cen J, Roomp K et al (2018) Identification of early biological changes in palmitatetreated isolated human islets. BMC Genom 19:1-11. https://doi.org/10.1186/s12864-018-5008-z

62. Chen $\mathrm{Q}$, Xiong $\mathrm{C}$, Jia $\mathrm{K}$ et al (2019) Hepatic transcriptome analysis from HFD-fed mice defines a long noncoding RNA regulating cellular cholesterol levels. J Lipid Res 60:341-352.

https://doi.org/10.1194/jlr.M086215

63. Hegardt FG (1998) Transcriptional regulation of mitochondrial HMG-CoA synthase in the control of ketogenesis. In: Biochimie

64. Köster A, Chao YB, Mosior M et al (2005) Transgenic angiopoietin-like (Angptl)4 overexpression and targeted disruption of Angptl4 and Angptl3: Regulation of triglyceride metabolism. Endocrinology. https://doi.org/10.1210/en.2005-0476

65. Tencomnao T, Yu RK, Kapitonov D (2001) Characterization of the human UDP-galactose: ceramide galactosyltransferase gene promoter 1. 1517:416-423

66. Cook ECL, Nelson JK, Sorrentino V et al (2017) Identification of the ER-resident E3 ubiquitin ligase RNF145 as a novel LXR-regulated gene. PLoS One. https://doi.org/10.1371/journal.pone.0172721

67. Koul O, Chou KH, Jungalwala FB (1980) UDP-galactose-ceramide galactosyltransferase in rat brain myelin subfractions during development. Biochem J. https://doi.org/10.1042/bj1860959 
68. Schaeren-Wiemers N, Van Der Bijl P, Schwab ME (1995) The UDP-Galactose:Ceramide Galactosyltransferase: Expression Pattern in Oligodendrocytes and Schwann Cells During Myelination and Substrate Preference for Hydroxyceramide. J Neurochem. https://doi.org/10.1046/j.1471-4159.1995.65052267.x

69. Zhang L, Rajbhandari P, Priest C et al (2017) Inhibition of cholesterol biosynthesis through RNF145dependent ubiquitination of SCAP. Elife. https://doi.org/10.7554/eLife.28766

70. Menzies SA, Volkmar N, van den Boomen DJH et al (2018) The sterol-responsive RNF145 E3 ubiquitin ligase mediates the degradation of HMG-CoA reductase together with gp78 and hrd1. Elife. https://doi.org/10.7554/eLife.40009

71. Sato C, Black JA, Yu RK (1988) Subcellular Distribution of UDP-Galactose:Ceramide Galactosyltransferase in Rat Brain Oligodendroglia. J Neurochem. https://doi.org/10.1111/j.14714159.1988.tb02493.x

72. Sprong H, Kruithof B, Leijendekker R et al (1998) UDP-galactose:ceramide galactosyltransferase is a class I integral membrane protein of the endoplasmic reticulum. J Biol Chem. https://doi.org/10.1074/jbc.273.40.25880

\section{Figures}


a

MAP2

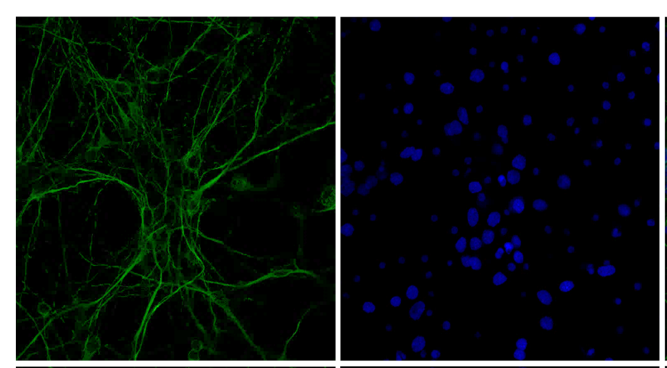

Control

$100 \mu \mathrm{M}$ PA

$300 \mu \mathrm{M}$ PA

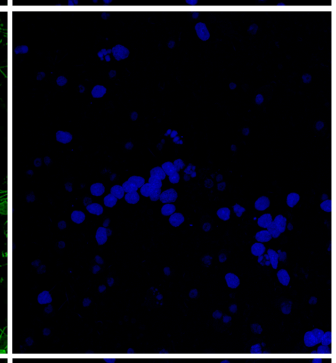

Merge
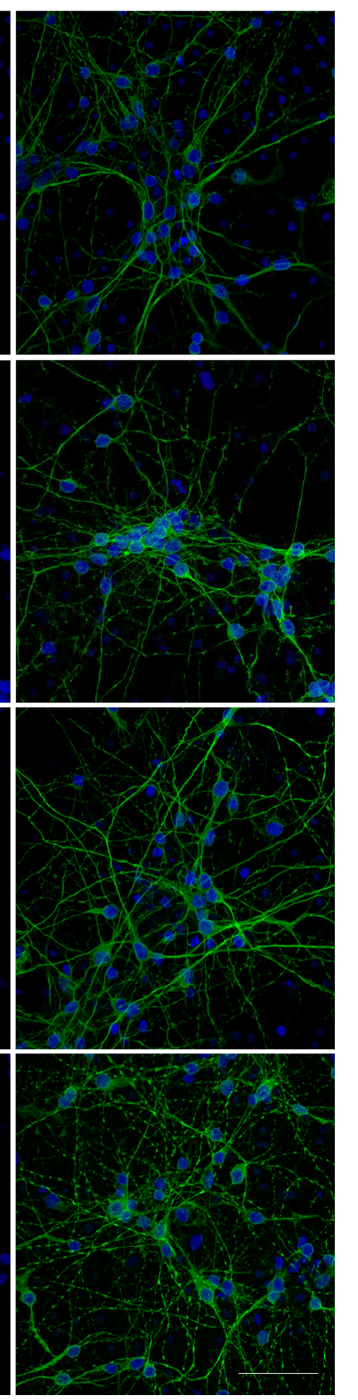

b

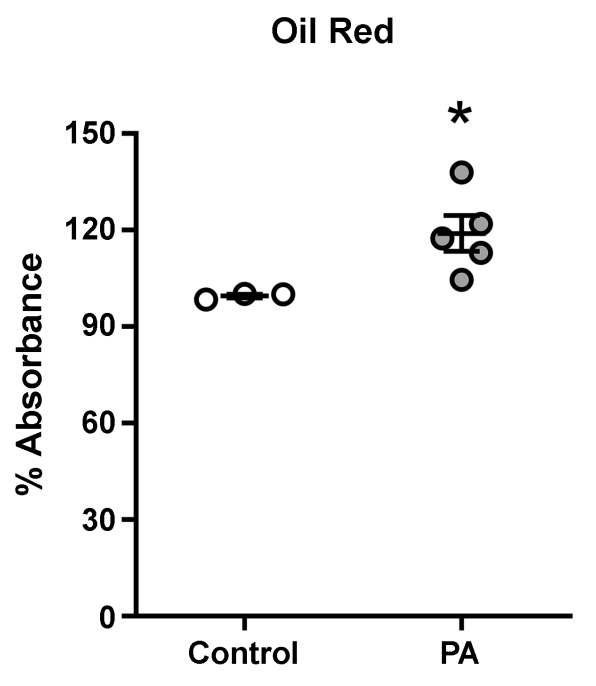

\section{Figure 1}

Palmitic acid-induced changes on neurites morphology and increases the lipid body content in cultured hippocampal neurons. a Representative images of MAP2 immunolabelling (green) and DAPI stained nuclei (blue) obtained with confocal microscopy showing neurites morphology in cultured hippocampal neurons treated for $24 \mathrm{~h}$ with vehicle, $100 \mu \mathrm{M}, 200 \mu \mathrm{M}$ and $300 \mu \mathrm{M}$ PA. For each panel confocal images were obtained with a 60x objective. b Quantification of Oil Red staining by spectrophotometry. Treatment for 24 hours with PA $200 \mu \mathrm{M}$ raises the content of lipid bodies compared to control conditions. * $\mathrm{p} \leq 0.005$. $\mathrm{N}=3$ 
a

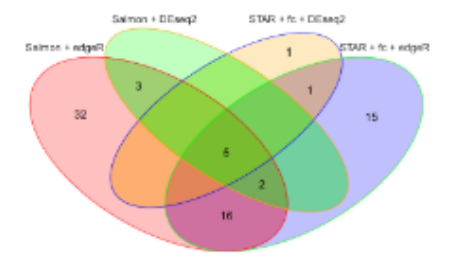

b

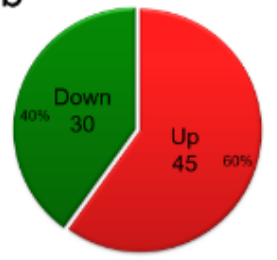

d

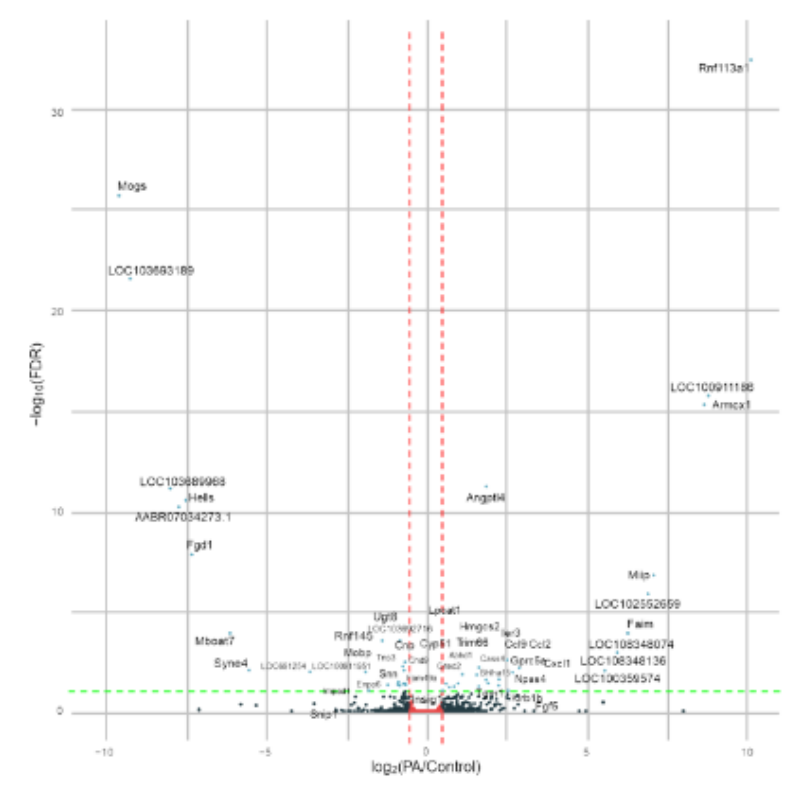

C

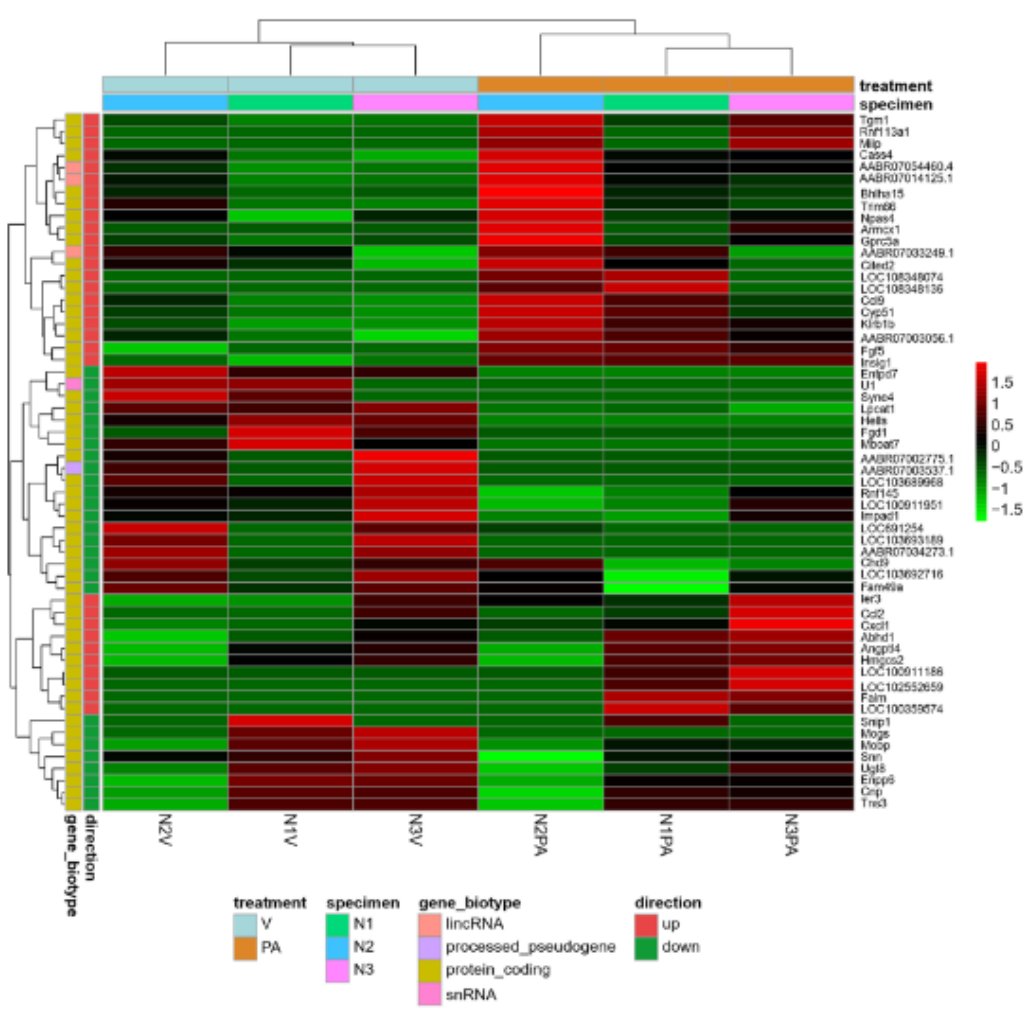

Figure 2

Transcriptome analysis of hippocampal neurons exposed to PA. a Number of DEG found with the RNAseq employing different pipelines. b Pie chart depicting the total number and percentage of differentially down- and upregulated genes found by RNAseq analysis. c Heat map showing the clustering of control group (V) vs. the PA exposed hippocampal neurons. $d$ Volcano plot of DEG. Genes not significantly different are depicted by dark-colored dots 
a GO term enrichment

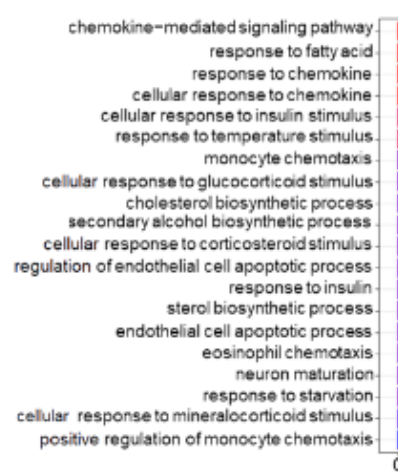

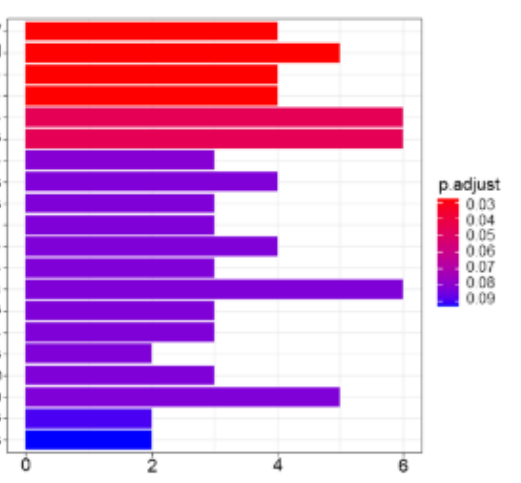

b

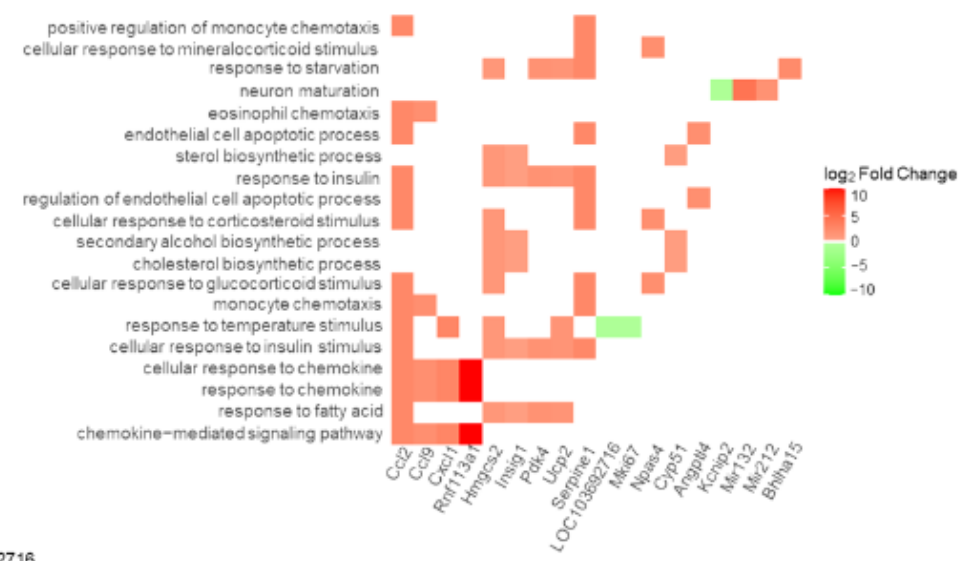

LOC1036927 16
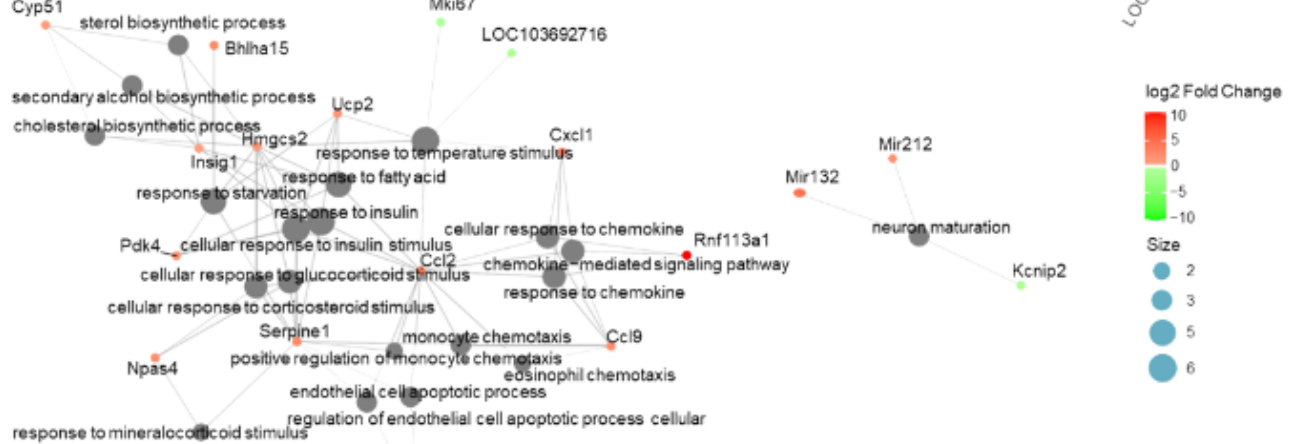

Angpt14

\section{Figure 3}

GO term enrichment analysis reveals metabolic and inflammatory processes altered in neurons after PA exposure. a Top 20 biological processes that were found to be significantly enriched (hypergeometric test FDR < 0.1) in response to PA exposure. b Heatmap of genes contained in the GO terms, each square represents the fold change of individual genes ( $x$-axis) and to which biological processes ( $y$-axis) they are associated with. c Interaction network of genes and GO terms, where gray nodes represent pathways and the green to red color-scaled nodes are DE genes linking or associating them 
a
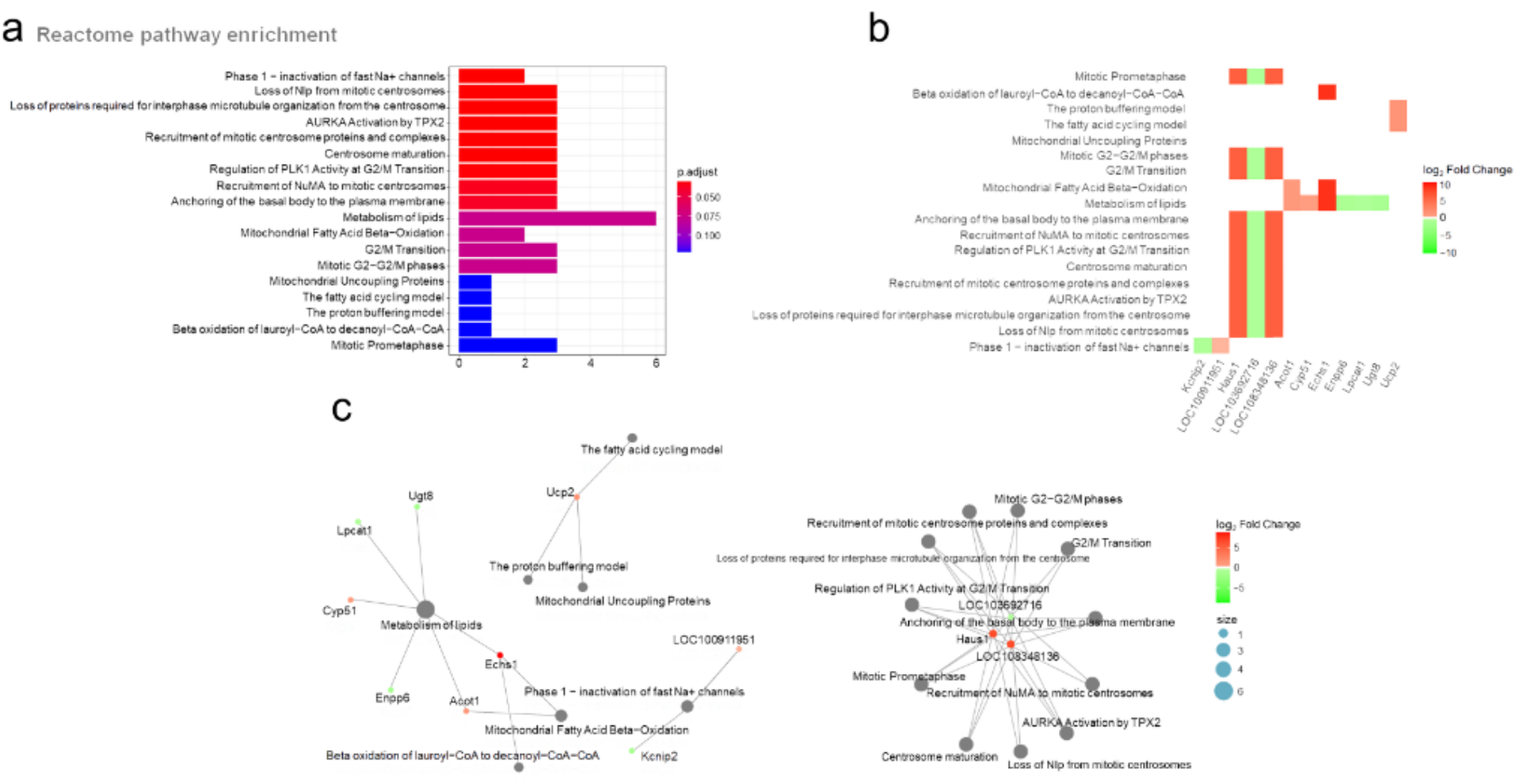

\section{Figure 4}

Reactome pathway enrichment analysis reveals metabolic and lipid processes altered in neurons when exposed to PA. a Top Reactome pathways that were found to be significantly enriched (hypergeometric test FDR < 0.1 ) in response to PA exposure. b Heatmap of genes contained in the GO terms. Each square represents the fold change of individual genes ( $x$-axis) and to which pathways ( $y$-axis) it is associated with. c Interaction network of genes and pathways, where gray nodes represent pathways and the green to red color-scaled nodes are DE genes linking or associating them 


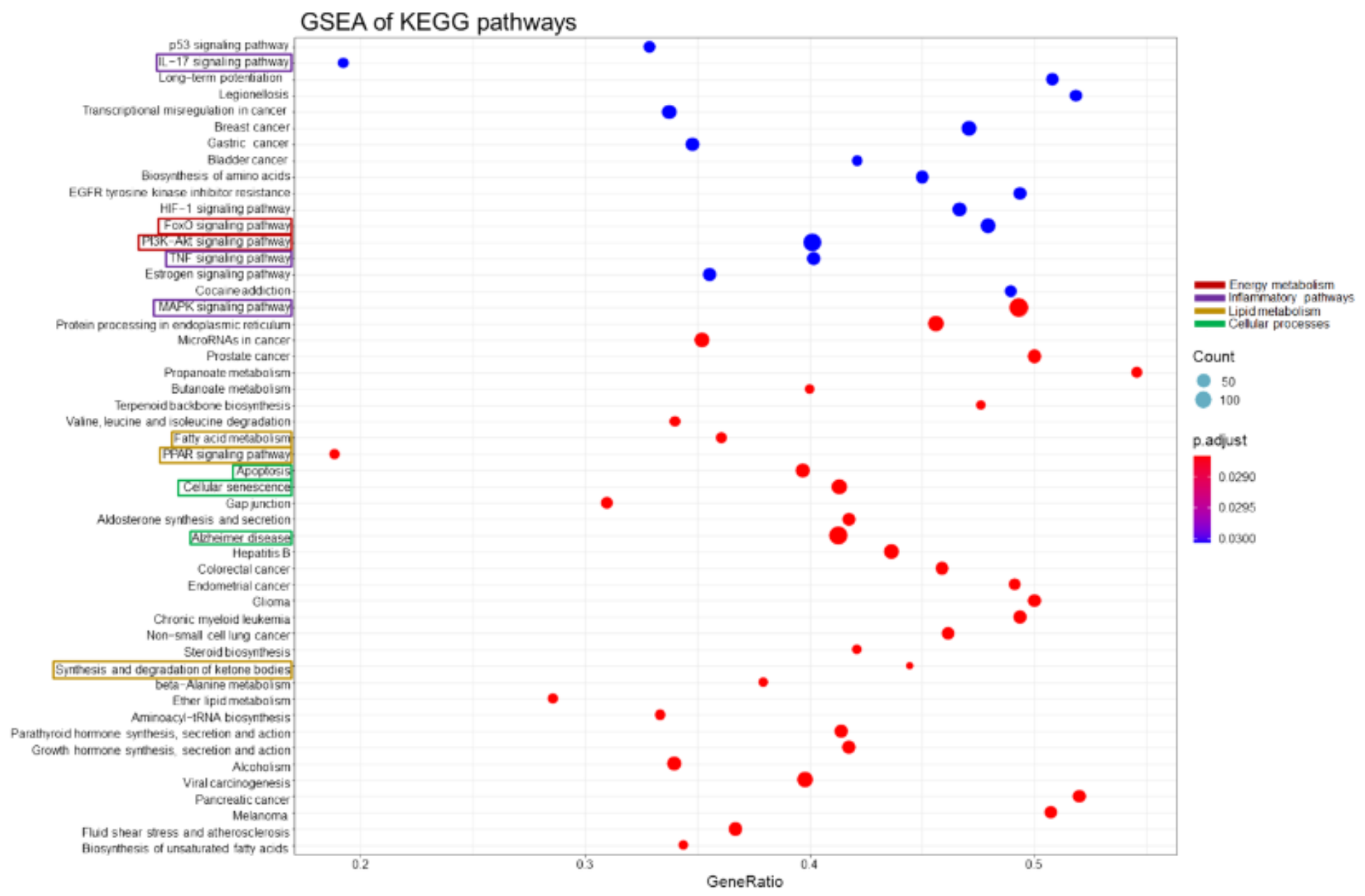

Figure 5

Gene Set Enrichment Analysis (GSEA) of KEGG. The top pathways and biological processes analyzed by KEGG (y-axis) and the number of genes involved in each process that are altered ( $x$-axis) are depicted in the graph. The affected metabolic pathways (red rectangles), lipid processes (yellow rectangles), immunological pathways (purple rectangles) and other important biological and cellular processes (green rectangles) when neurons are exposed to PA are highlighted 
a
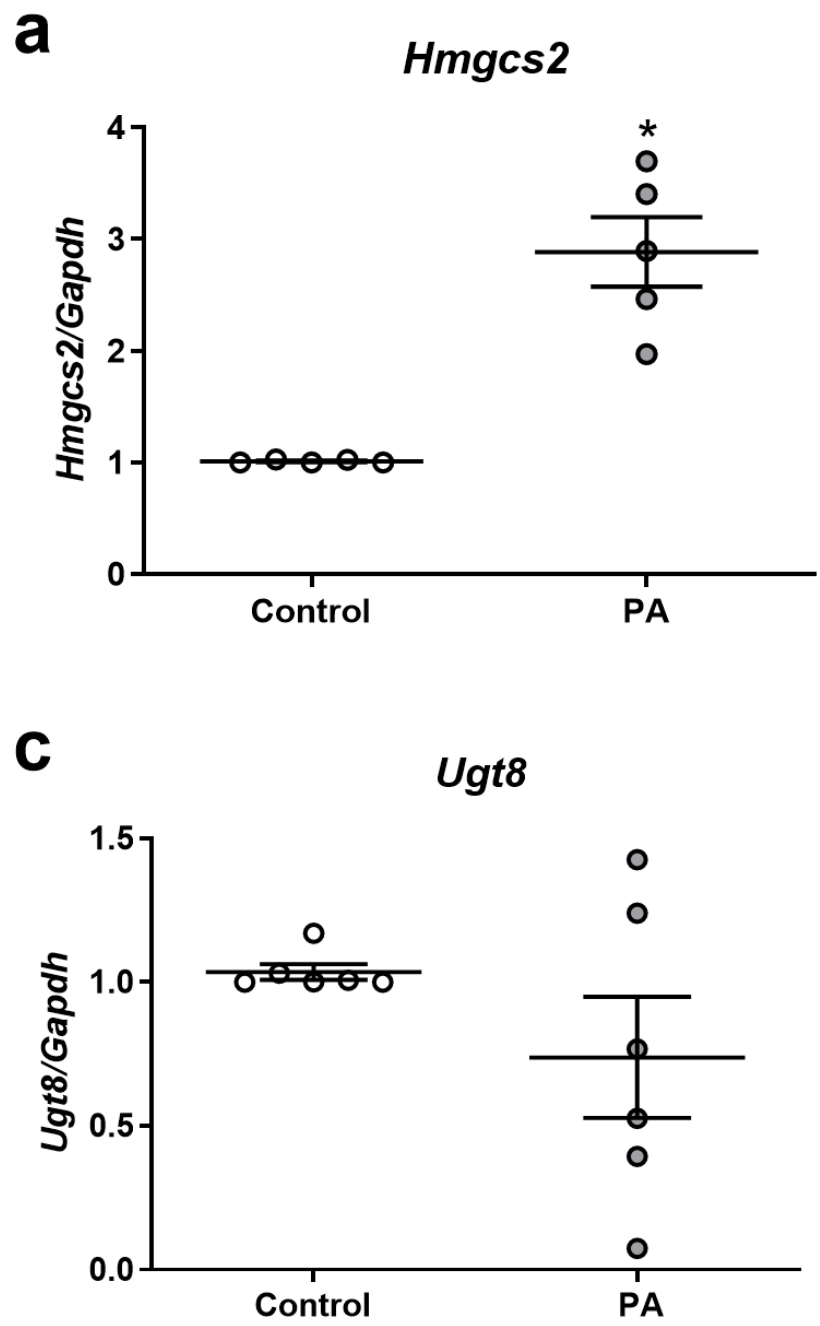

b

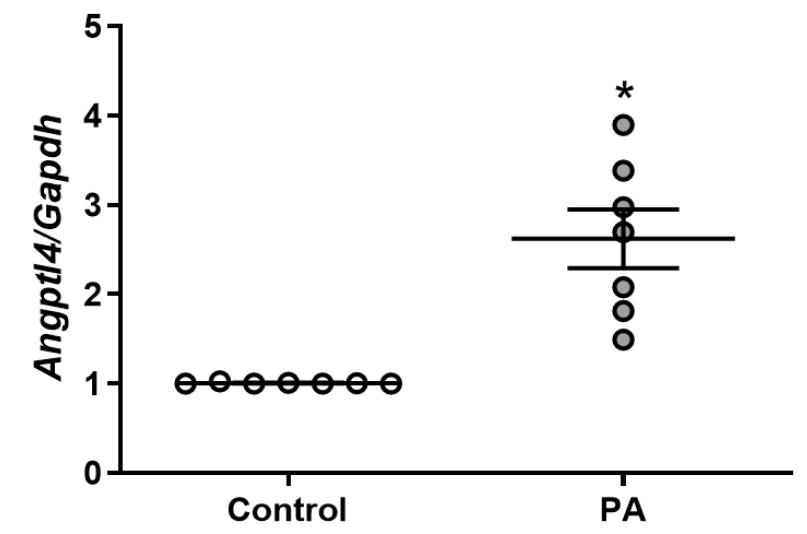

d

Rnf145

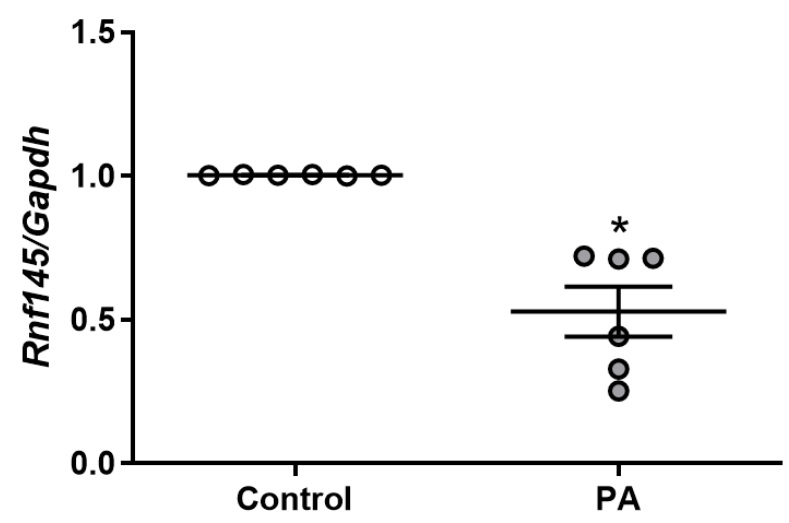

Figure 6

Expression levels of Hmgcs2, Angptl4, Ugt8 and Rnf145 after 24 hours of $200 \mu \mathrm{M}$ PA treatment. The analysis of expression by qPCR shows that when neurons are exposed to $200 \mu \mathrm{M}$ PA, both a Hmgcs2 and b Angpt|4 are overexpressed, while both c Ugt8 and d Rnf145 are downregulated compared to the control group. Biological replicates were performed at least five times with ${ }^{*} \mathrm{p} \leq 0.005$

\section{Supplementary Files}

This is a list of supplementary files associated with this preprint. Click to download.

- Supplementarylnformation.pdf 